\title{
Elemente einer ethischen Grundrechtstheorie
}

\author{
Christoph Möllers"
}

Rezension zu: Matthias Mahlmann, Elemente einer ethischen Grundrechtstheorie, Baden-Baden (Nomos ff Neue Schriften zum Staatsrecht Band 3), 2008, 553 S., $109 €$, ISBN 978-3-8329-1634-3

1. Die Suche nach einer verfassungsadäquaten Theorie des Grundgesetzes oder zumindest seines Grundrechtsteils begleitet unsere Verfassung seit ihrer Entstehung, wenn sie auch erst in zwei berühmten Beiträgen Ernst-Wolfgang Böckenfördes aus den 1970er Jahren zu einem ausdrücklichen wissenschaftlichen Desiderat erklärt wurde.

Diese Suche bringt ein Dilemma zum Ausdruck, allerdings eines, auf das einzulassen sich lohnt, wenn es nicht für eine ernsthafte Rechtswissenschaft sogar unvermeidlich ist: Denn wenn eine grundgesetzadäquate Theorie mehr sein will, als das Grundgesetz vorgibt, dann wird sich die Frage nach der Rechtfertigung des Anspruchs stellen, den die Theorie erhebt, uns das Grundgesetz zu erklären. Wenn sich die Theorie dagegen darauf beschränkt, uns unbestrittene Inhalte des Grundgesetzes vorzustellen, kann man auch auf sie verzichten.

Dass dieses Dilemma die Verfassungsrechtswissenschaft nicht ernsthaft daran hindern sollte, sich auf die Suche nach einer entsprechenden Theorie zu machen, liegt schon daran, dass wir gar keine Vorstellung davon haben könnten, was wir meinen, wenn wir vom „Grundgesetz“ sprechen, ohne damit zugleich theoretische Voraussetzungen zu verbinden. Es geht also gar nicht darum, eine Theorie des Grundgesetzes zu haben oder nicht; es geht vielmehr darum, ob man die Theorie, die man ohnehin hat, ausdrücklich macht oder ob man sie verdeckt. Nur, wenn man sie ausdrücklich macht, kann man sie aber auch zur wissenschaftlichen Diskussion stellen, nur wer seine Theorie offenbart - und begründet - nimmt an einem wissenschaftlichen Dialog über das Grundgesetz teil - und bastelt nicht nur in der Praktikerwerkstatt. Die systematische Verdunkelung dieses Umstandes durch eine unglückliche Kombination aus Verfassungsgerichtsfixiertheit und Methodennaivität, die es manchmal sogar so weit bringt, die „vier Auslegungsmethoden“ als angemessenes Werkzeug für die Grundgesetzexegese zu empfehlen, gehört vielleicht zu den größten Versäumnissen der heutigen Ausbildung im Verfassungsrecht.

Schon aus diesem Grund wird man mit Spannung das vorliegende Buch öffnen, das sich die Entwicklung eines theoretischen Vorverständnisses des Grundrechtsteils des Grundgesetzes zum Ziel gesetzt hat. Mahlmanns Schrift, eine Habilitationsleistung

* Prof. Dr.Christoph Möllers LL.M. lehrt Öffentliches Recht, insbesondere Verfassungsrecht, und Rechtsphilosophie an der Juristischen Fakultät der Humboldt-Universität zu Berlin. 
an der Juristischen Fakultät der Freien Universität Berlin, ist sich aller methodischen Schwierigkeiten des eigenen Projekts voll bewusst und geht sie in einer instruktiven Einleitung geradlinig an.

Dass ein solches Projekt trotzdem immer unter dem Problem leiden muss, sich selbst Grenzen zu ziehen, wird freilich auch in dieser Einleitung schon deutlich. In Bezug auf Böckenfördes Verständnis der Verfassung als „Rahmenordnung“ bemerkt der Verfasser dort: „Auch die Idee der Rahmenordnung befreit im Übrigen nicht von der Aufgabe, den Inhalt dieses Rahmens zu bestimmen. “(S. 15). Diese Feststellung kann schwerlich richtig sein, würde dies doch die gesamte Metapher ihrer Bedeutung berauben. Es mag schlanke und dicke Rahmen geben, verzierte und karge, aber wenn der Rahmen nach innen kein Ende findet, ist er eben kein Rahmen mehr, sondern ein ungerahmtes Bild.

2. Mahlmanns Buch zielt auf eine „ethische“ Grundrechtstheorie, also auf eine Fundierung, die ihre Begrifflichkeiten und Wertungen der praktischen Philosophie entnimmt. Die Arbeit entwickelt sich zu diesem Zweck in vier großen Teilen, deren erster sich dem Verhältnis von Moral und Recht allgemein zuwendet, deren zweiter die Kategorie der Menschenwürde untersucht, deren dritter sich dem Verhältnis von Freiheit und Gleichheit und exemplarisch dem Verständnis der Allgemeinen Handlungsfreiheit und dem allgemeinen Gleichheitssatz des Grundgesetzes widmet und deren vierter und letzter Teil unter der nicht ganz aussagekräftigen Überschrift „Rationalität und ethische Grundrechtstheorie“ das Problem der Universalisierbarkeit von Grund- und Menschenrechten angeht.

Dies sind für sich große Brocken, und auch die feinere Einteilung des Buches in eine Einleitung und neun Kapitel kann nicht verhindern, dass sich der Leser schnell als Teilnehmer eines intellektuellen Rundflugs fühlt, auf dem man viel zu sehen bekommt, wenn auch aus einer Distanz, in der sich auch Dinge ähnlich zu sehen beginnen, die einem in anderen Zusammenhängen doch ganz verschieden vorkommen. Dies gilt zunächst für den großen ideengeschichtlichen Durchgang durch das Verhältnis von Recht und Moral von der Antike bis zur Gegenwart in Kapitel 1. Es gilt, wenn auch nur in abgeschwächter Form, gleichfalls für die folgende Darstellung der Rechtsprechung des Bundesverfassungsgerichts zur Menschenwürdegarantie und zur sie begleitenden wissenschaftlichen Diskussion. Diese Teile sind gut lesbar und argumentiert, aber vornehmlich zügig referierend, mitunter auch bewertend, allerdings ohne dass die Grundlagen dieser Bewertung an dieser Stelle schon nachvollziehbar geworden wären.

3. Erst in der Mitte von Kapitel 5 (S. 262 ff.) gewinnt die eigene Position Mahlmanns Kontur. Hier entwickelt er seine Konzeption der Menschenwürde, die den Anspruch erhebt, die Achtung der Menschenwürde aus der Perspektive einer „humanistischen 
Anthropologie" (kann man ein griechisches Substantiv durch ein lateinisches Adjektiv gleicher Bedeutung sinnvoll ergänzen?) zu begründen. An diesem Punkt (S. 264 f.) liegt vielleicht das entscheidende Element der gesamten Argumentation:

„Die Naturnotwendigkeit, sich selbst Zweck zu sein, besagt, dass es ein anthropologisches Faktum sei, dass der natürliche Zweck, den die Menschen sich setzen, das Gedeihen ibrer eigenen Person, das mögliche Entfalten ibrer Person zu einem Glück sei.“

und

„Da alle Menschen sich empirisch als Selbstzweck betrachten und keiner unter Gerechtigkeitsgesichtspunkten einen Selbstzweck vor anderen Menschen genießt, ist kein einzelner Mensch allein und gar auf Kosten anderer, sondern jeder Mensch gleichermaßen und damit die Menschen insgesamt und als Sammelbegriff der einzelnen Individuen ein Zweck an sich."

Mahlmann hält dieses Argument deswegen ausdrücklich nicht für einen Sein-SollensFehlschluss, weil das normative Gebot proportionaler Gleichbehandlung hier nur auf das Faktum der jeweiligen Selbstzweckhaftigkeit der Individuen angewendet werde. Hier scheint freilich einiges durcheinander zu gehen: Zum ersten ist die Frage der individuellen Selbstzweckhaftigkeit empirisch durchaus zweifelhaft. Individuen mögen gerade aus moralischen Motiven von sich selbst absehen und sich beispielsweise opfern. Diese Phänomene ließen sich jedenfalls nicht in eine Argumentation einbauen, die vorgibt empirisch zu sein. Zum zweiten lässt sich das normative Gebot proportionaler Gleichbehandlung, wenn man seine Geltung denn akzeptiert - und das versteht sich keineswegs von selbst (siehe dazu nur Jeremy Waldron, God, Locke, and Equality 2002) - kaum auf ein empirisches Faktum anwenden. Anerkennung entsteht nicht durch die Verallgemeinerung von Empirie. Wäre der Mensch dem Menschen ein Wolf, würde dann daraus in Anwendung des Gleichheitsprinzips eine Pflicht entstehen, sich gegenseitig aufzuessen? Und kann man schließlich, zum dritten, wirklich behaupten, dass die verallgemeinernde Normativierung eines Faktums keinen Sein-Sollens-Schluss darstellt?

Es mag heute, unter den Bedingungen einer evolutionsbiologischen Forschung, die uns viel über den Menschen berichtet, und die durchaus zu spezifizieren weiß, was ihn vor anderen Lebewesen auszeichnet, durchaus angemessen erscheinen, die Tradition der philosophischen Anthropologie in normativer Absicht wieder zu entdecken. Mahlmann, ein Autor, der stets naturwissenschaftlich gut informiert argumentiert, hätte diesen Versuch unternehmen können. Doch kann man nicht alles haben - und man kann sicherlich nicht eine gattungstheoretische Methode wie die Anthropologie anwenden und zugleich darauf Wert legen, das Verbot des naturalistischen Fehlschlusses zu beachten. 
Ein konsistentes Vorgehen hätte vielmehr die Bedeutung und die Berechtigung dieses Verbots in Frage stellen müssen, wie es sich ja etwa aus der aristotelischen Tradition gut begründen lässt. Wenn andererseits die ganze „Empirie“, mit der das entscheidende Faktum begründet werden soll, in einer Uminterpretation der Kantischen Moralphilosophie besteht, wie es in den zitierten Abschnitten der Fall ist, dann handelt es sich eben doch um eine rein begriffliche Konstruktion, die den deskriptiven Anspruch der Anthropologie nicht ernst nimmt.

Dieses methodische Problem des Buches lässt sich auch an von einem anderen Blickwinkel aus beleuchten: Seinen Anspruch, eine ethische Theorie der Grundrechte zu entwickeln, dürfte man aus Sicht der praktischen Philosophie mit der Frage begegnen, welche Ethik denn gemeint sei. Mahlmann beantwortet diese Frage mit einer Definition:

„Moral ist eine subjektive, inhaltich wesentlich am Guten und an Gerechtigkeit orientierte, durch Urteilsakte im Gewissen der einzelnen Menschen gebildete, durch den individuellen Willen unmittelbar affizierende Sollensgebote verbindliche, jedenfalls psychisch sanktionierte, spezifische Gefüble bedingende Ordnung. "(S. 76, kursiv i.O.).

Wenig später greift er den Begriff des Wertes auf, um festzustellen, dass sein Projekt einer ethischen Grundrechtstheorie die materialen Einflüsse von moralischen Werten auf die Grundrechtsausübung reflektiert (S. 92).

Schon die erste Definition erscheint als solche zumindest anfechtbar, jedenfalls ist sie Gegenstand einer zentralen Auseinandersetzung innerhalb der gegenwärtigen metaethischen Debatte, die gerade die Frage aufwirft, ob es zum Begriff der moralischen Verpflichtung gehört, ein internes Handlungsmotiv zu stiften. Diese Position ist sicherlich gut vertretbar, aber sie ohne Angaben einer Diskussion in einer Definition als gegeben zu unterstellen, erscheint eigenartig. Zudem ist die strikt empirische Formulierung des Motivs nicht selbstverständlich; wenn Moral bestimmte „Gefühle“ erzeugt, dann dürfte es schwierig sein, diese subjektive Wirkung in ein Konzept von Willensfreiheit zu integrieren - wie es Mahlmann doch im Folgenden ausdrücklich versuchen wird.

Diese Bedenken verstärken sich zusätzlich, wenn man die zitierte Wendung zum Wertbegriff einbezieht; denn wenn der Begriff „Wert“ irgendeine theoretische Kontur haben soll, dann dürfte diese in seiner Objektivität bestehen. Ein moralischer Wert verliert seine normative Qualität gerade nicht dadurch, dass er mich als Subjekt nicht beeindruckt.

Man mag in diesen Inkonsequenzen eine typisch begriffsökumenische Argumentationsstrategie erkennen, die sich gerade in interdisziplinär argumentierenden juristi- 
schen Arbeiten oftmals findet: interne Diskussionen der anderen Disziplin können hier bis zu einem gewissen Grad offenbleiben. In Mahlmanns Buch stellen sie freilich, wie wir gesehen haben, den entscheidenden Kern seiner eigenen Argumentation dar, und nicht nur eine Absicherung gegenüber einem Nachbardiskurs. Denn sowohl die Herleitung der Menschenwürde aus einem anthropologischen Rechtsverständnis als auch sein allumfassender Begriff von Moral sind wichtige Elemente seiner Konzeption.

4. Im Fortgang der Argumentation entwickelt Mahlmann auf dieser Grundlage eine eigene Dogmatik der Menschenwürde, die man als systematische Weiterentwicklung der vom Bundesverfassungsgericht verwendeteten Dürig'schen Objektformel verstehen kann. In der Anwendung dieser Grundlegung auf einzelne Probleme, namentlich der Bioethik, erweist sich die Darstellung als durchaus differenziert und geeignet, die Diskussion weiter zu bringen. Seine Argumentation illustriert auch noch einmal die Richtigkeit seines Ausgangspunktes, der Einsicht, dass eine grundrechtliche Argumentation ohne ein theoretisches Vorverständnis nicht auskommt.

Weniger dramatisch, aber auch weniger originell verlaufen die beiden folgenden Kapitel 6 und 7 über die Freiheits- und Gleichheitsrechte des Grundgesetzes, die im Prinzip eine affirmative Rekonstruktion herrschender Lehren anbieten. Die Arbeit nimmt die neueste Runde der grundrechtsdogmatischen Diskussion um die Bestimmung von grundrechtlichen Schutzbereichen leider nicht auf.

In den beiden letzten Kapiteln des Buches wendet sich Mahlmann noch einmal dem Problem des Relativismus zu. Entsprechend der anthropologischen Anlage seiner Grundrechtstheorie kann er die Ergebnisse seiner Arbeit durchaus als universal ausweisen. Kapitel 8 untersucht dazu die Möglichkeit einer allgemeinen Theorie der Sprache, die es ausschließen soll, die notwendige sprachliche Vermittlung von Normativität zum Anlass zu nehmen, kulturelle Eigenheiten unterschiedlicher Grundrechtsordnungen zu postulieren.

Das Schlusskapitel kritisiert deshalb andere rechtstheoretische Ansätze, die gegenüber diesem Anspruch skeptisch bleiben. An dieser Stelle bleibt die Argumentation freilich relativ pauschal, exemplarisch sei auf die Diskussion der Wittgenstein'schen Sprachphilosophie hingewiesen (S. 468 f.), eines der meist debattierten philosophischen Probleme der zweiten Hälfte des 20. Jahrhunderts, das hier weder wirklich ausgeführt noch entsprechend zugespitzt wird. Entsprechend der Gesamtlinie des Buches begründet das abschließende Kapitel angesichts der neu entstehenden Systeme des internationalen Menschenrechtsschutzes die Erwartung an das Entstehen einer gemeinsamen internationalen Menschenrechtskultur. Inwieweit diese Erwartung berechtigt ist, insbesondere inwieweit die doch immensen Varianzen zwischen den verschiedenen nationalen und internationalen Menschenrechtssystemen nur als eine 
Art technischer Panne verstanden werden müssen und nicht doch auch als Ausdruck einer legitimen, aber widerspruchsreichen Vielfalt, ließe sich letztlich nur durch vergleichende Stichproben zeigen. Die Hoffnung auf eine internationale Menschenrechtskultur gründet auf einem Postulat oder auf Spekulation.

5. Mahlmanns Buch baut an der ganz großen Konvergenzthese, in der Anthropologie und Menschenwürde, Freiheit und Vernunft ebenso zusammenfinden wie die Unterscheidung zwischen Deskriptivem und Normativem. Der Sprung von einem, wenn auch recht inkonsequent durchgeführten, Naturalismus eines anthropologischen Arguments zur Auslegungspraxis des Grundgesetzes kennt in seiner Rekonstruktion keine anderen Vermittlungsstufen, insbesondere keinen Raum für kulturelle Differenzen.

Dass viele westliche Rechtsordnungen mit einer älteren freiheitlichen Tradition von einer Menschenwürdegarantie ebenso wenig gehört haben wie von einem flächendeckenden Grundrechtsschutz durch eine allgemeine Handlungsfreiheit, kann den Verfasser da wenig anfechten, wenn er dieses überhaupt zur Kenntnis nimmt. $\mathrm{Zu}$ ihrer eigentlichen Bestimmung scheint die Gattung Mensch erst durch die Grundrechtsinterpretation des Bundesverfassungsgerichts gefunden zu haben. Der Schutz der Grundrechte, insbesondere der Menschenwürde, ergibt sich aus der richtigen wissenschaftlichen Beschreibung der conditio humana - und diese kennt für historische, politische oder andere kulturelle Kontingenzen ganz ausdrücklich keinen Raum. Wer dies bestreitet, etwa weil er ein postmoderner Relativist ist, irrt.

Man wird den Charme dieses radikalen Standpunktes und die Geschicklichkeit, mit der Mahlmann ihn entwickelt, auch dann anerkennen können, wenn man ihn im Ergebnis nicht teilt. Über die Konsequenzen einer Position, die zwischen politischem Dissens und moralischer Verwerflichkeit wohl nicht mehr zu unterscheiden vermag, muss sich eine wissenschaftliche Arbeit wohl auch nicht bekümmern.

Wissenschaftlich bedenklicher ist die notwendige Unschärfe, die der Zugang mit sich bringt; denn sehr vieles wird in diesem Buch angesprochen und immer wird - dies ist keine geringe Leistung - eine bestimmte Linie und eine bestimmte Sicht auf die angesprochenen Phänomene durchgehalten; doch werden eben auch fast alle dargestellten Positionen - vom Würdeverständnis der Antike bis zur Menschenwürdediskussion unter dem Grundgesetz, von der Bedeutung des internationalen Menschenrechtsschutzes bis zur Philosophie der Postmoderne - sehr verknappt, wenn nicht verzeichnet dargestellt. Am Ende scheint der Text eher durch ein Postulat als durch ein Argument zusammengehalten zu werden. Wiederum positiv gewendet handelt es sich beim vorliegenden Buch um eine Synthese, die uns deutlich macht, was Universalität der Grundrechte bedeutet und welcher methodische Preis dafür zu bezahlen ist, an diese Universalität zu glauben. 